\title{
I've got you under my skin
}

The proteasome inhibitor bortezomib has increased the overall survival of patients with multiple myeloma, becoming the standard of care for this disease. However, bortezomib has two downsides: the first one is its adverse effects (mainly peripheral neuropathy), and the second one is its via of administration (intravenous and twice per week). This form of route of administration is stressful for patients, who have to visit the hospital relatively frequently, and can also be painful, especially for those patients who have poor venous access. For these reasons, Moreau and collaborators were determined to optimize the use of bortezomib, by testing the efficacy and safety of administrating this drug subcutaneously.

To this end, the researchers led a multicenter phase III study that included 222 patients, 148 of whom were assigned to receive a median of eight cycles of bortezomib subcutaneously, and 74 of whom received the same dose intravenously. In both groups, those patients with a suboptimun response after cycle four also received dexamethasone. Subcutaneous administration proved to be as efficient as the intravenous administration: response rates, time to first response, and duration of response were similar between the two groups and, after 1 -year follow-up, there was no significant difference in time to progression either.

Regarding safety, subcutaneous administration of bortezomib seemed to have lower rates of peripheral neuropathy (38\% versus $53 \%$ ) and other adverse events (57\% versus 70\%). Moreover, bortezomib was well tolerated when administered subcutaneously, offering the possibility of home administration that would improve patient convenience.

\section{Teresa Villanueva}

Original article Moreau, P. et al. Subcutaneous versus intravenous administration of bortezomib in patients with relapsed multiple myeloma: a randomised, phase 3 , non-inferiority study. Lancet Oncol. 12, 431-440 (2011) 